\title{
CHARACTERISTICS OF PULSE COMPRESSION IN LASER PULSE AMPLIFICATION BY STIMULATED RAMAN BACKSCATTERING*
}

\author{
Jincheol B. F. Kim ${ }^{\mathrm{a}, \mathrm{b}}$, Hae June Lee ${ }^{\mathrm{b}}$, Hyyong Suk ${ }^{\mathrm{b}}$, In Soo Ko ${ }^{\mathrm{a}}$ \\ ${ }^{a}$ Department of Physics, POSTECH, San-31, Hyoja-dong, Pohang, Kyoung-buk, \\ 790-784, South Korea \\ ${ }^{\mathrm{b}}$ Center for Advanced Accelerators, Korea Electrotechnology Research Institute, \\ Seongju-dong 28-1, Changwon, 641-120, South Korea
}

\section{Abstract}

The characteristics of pulse compression during the laser pulse amplification using a counter-propagating pump and plasmas have been investigated using a onedimensional fluid model of stimulated Raman backscattering. The pulse widths of the amplified laser at the front and rear half are examined in terms of the initial amplitudes of the pump and the seed, and the ratio of the electron plasma frequency to the laser frequency. The pulse widths have a common scaling behaviour in time over the considered parameter regime. The scaling exponents of the pulse widths during broadening and compression are all the same as 1.0. The pulse compression scaling shows a reciprocal behaviour to the peak energy growth.

\section{INTRODUCTION}

It was suggested that a short laser pulse (seed) of low energy can be amplified with a counter-propagating long laser pulse (pump) and a plasma by stimulated Raman backscattering (RBS) [1-4]. In this paper, we present simulation results on the characteristics of pulse compression during the amplification.

\section{ONE-DIMENSIONAL FLUID MODEL}

One-dimensional (1D) fluid model was used for the study of seed pulse amplification by RBS. The model follows the formulation of RBS in [1] and assumes a cold plasma. We do not consider the detuning term in this model. The governing equation for RBS for the case is

$$
\begin{aligned}
& \frac{\partial a_{p}}{\partial t}-v_{g, 0} \frac{\partial a_{p}}{\partial x}=\frac{c}{4} k_{f} \frac{\omega_{p}}{\omega_{0}} a_{s} f, \\
& \frac{\partial a_{s}}{\partial t}+v_{g, 1} \frac{\partial a_{s}}{\partial x}=-\frac{c}{4} k_{f} \frac{\omega_{p}}{\omega_{1}} a_{p} f^{*}, \\
& \frac{\partial f}{\partial t}-v_{g, 2} \frac{\partial f}{\partial x}+\Gamma f+i \frac{3}{8} \omega_{p}|f|^{2} f=-\frac{c}{4} k_{f} a_{s} * a_{p},
\end{aligned}
$$

where $f=e E_{x} / m c \omega_{p}$ is the normalized longitudinal electric field, and $a_{s}=e E_{1} / m c \omega_{1}$ and $a_{p}=e E_{0} / m c \omega_{0}$ are the normalized electric fields for the seed and the pump, respectively. $\omega_{j}$ and $k_{j}$ meet the matching condition, $k_{f}=$ $k_{0}+k_{l}$ and $\omega_{0}=\omega_{1}+\omega_{p}$, where $\omega_{j}$ and $k_{j}$ are the angular frequency and the wave number of the pump $(\mathrm{j}=0)$ and the seed $(\mathrm{j}=1)$, respectively. $v_{g, j}=c^{2} k_{j} / \omega_{j}$ is the group velocities for the seed $(j=1)$ and the pump $(j=0)$. In this study, we neglect the damping term and the relativistic nonlinear detuning term since $|f|$ is much smaller than 1 .
A moving window was used to make computations faster. The moving window is on the frame of the seed and advances with the speed of $v_{g, 1}$. The size of the moving window is about $500 \mu \mathrm{m}$ to $2000 \mu \mathrm{m}$. The used grid size $(\Delta x)$ ranges from 0.2 to $0.4 \mu \mathrm{m}$. It is small enough to observe the essential behaviour of 1D fluid model using a quasi-static eikonal approximation to analyze the slowly varying amplitudes. The confronting boundary condition of the moving window to the pump is the initial amplitude of the pump $\left(a_{p 0}\right)$. The trailing boundary condition at the end of the moving window is just to discard the previous quantities as ordinary moving window simulations do.

The initial spatial profile was set up in the moving window as the situation when the collision between the seed and the pump starts. It means that the interaction of the seed or the pump only with a plasma is negligible, which is valid for a non-relativistic low intensity laser pulses.

The time step is determined from the spatial grid size as $\Delta t=\zeta\left(\Delta x / v_{g, l}\right)$ with an appropriate complement factor $\zeta$, which is typically close to 1.0 .

We investigated the time evolutions of the full width at the half maximum (FWHM), the peak energy, and the widths of the front and rear half of the seed, $D_{f}$ and $D_{r}$ respectively. The wavelength of the pump $\left(\lambda_{0}\right)$ was set to be $0.532 \mu \mathrm{m}$. The seed and the plasma wavelengths were set to meet the matching condition. The parameter $\omega_{p} / \omega_{1}$ ranges from 0.02 to 0.25 by changing the plasma density. The initial pump amplitude, $a_{p 0}$, has a value from 0.003 to 0.020 which corresponds to the laser intensities of 40 $\mathrm{TW} / \mathrm{cm}^{2}$ and $2 \mathrm{PW} / \mathrm{cm}^{2}$ with $\lambda_{0}=0.532 \mu \mathrm{m} . a_{p 0}$ can be adjusted by changing the laser intensity or spot sizes in experiments. The pump is assumed to be a long square pulse with a pulse length $\left(\tau_{p}\right)$ of $20 \mathrm{ps}$. The initial seed amplitude was chosen to be from 0.001 to 0.020 . The seed was assumed to be a Gaussian pulse in time domain, $\exp (-$ $t^{2} / \tau_{s}^{2}$ ), where $\tau_{s}$ is from 0.2 to $1.0 \mathrm{ps}$. The initial FWHM is $1.18 \tau_{s}$.

\section{SIMULATION RESULTS}

\section{Linear and Nonlinear Growth Regime and Pulse Compression}

Figure 1 shows the time evolutions of the peak energy of the seed and the compression of the seed during the amplification. It is known that the peak energy grows in two stages. First, the seed is amplified exponentially, 
which is classified as a linear regime. In this regime, the seed broadens and the semi-log plot of the peak energy vs. time shows linear behaviour [Fig. 1(a)]. After the linear regime, the peak energy grows as $t^{\beta}$, which is classified as a nonlinear regime. The seed is compressed simultaneously in the nonlinear regime. The difference between the linear and the nonlinear regimes is associated with the depletion of the pump. The start of the nonlinear regime corresponds to the local depletion of the seed, which is followed, by the $100 \%$ depletion of the pump.

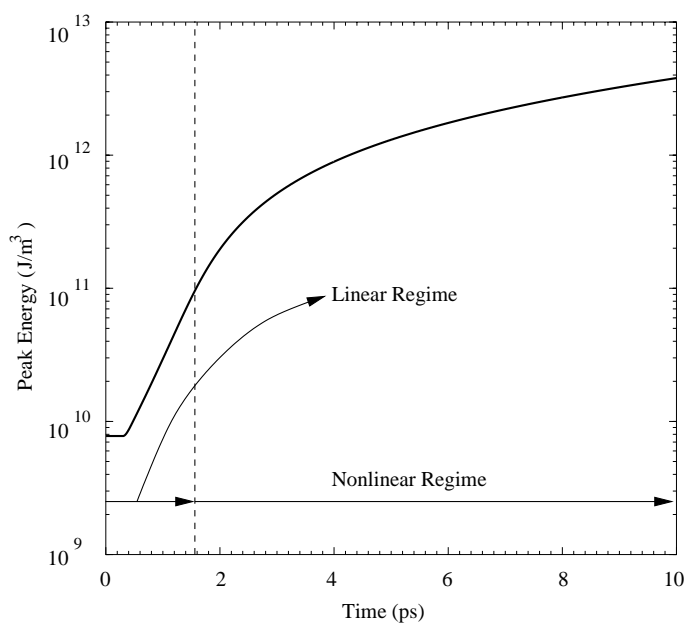

(a)

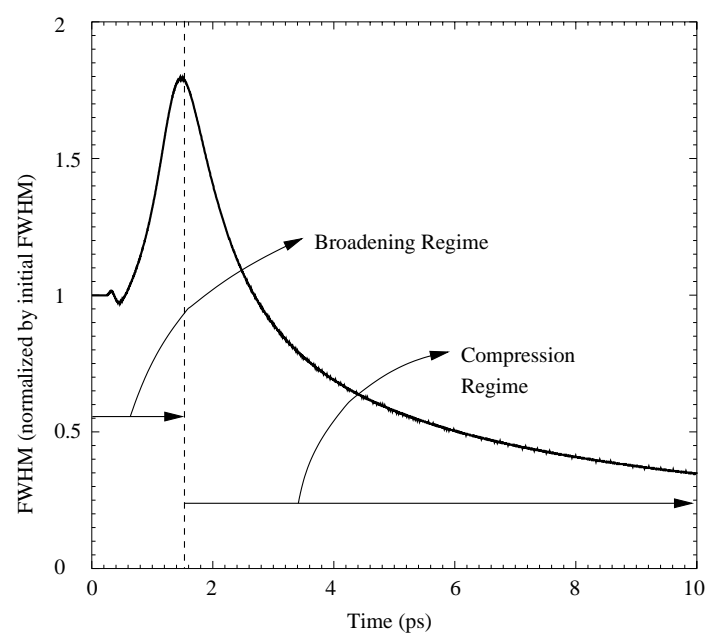

(b)

Figure 1: Time evolutions of (a) the peak energy and (b) the FWHM showing the linear and nonlinear regime of the seed amplification $\left(a_{p 0}=0.01, a_{s 0}=0.001, \omega_{p} / \omega_{1}=\right.$ $0.002, \tau_{p}=20 \mathrm{ps}, \tau_{s}=0.2 \mathrm{ps}$ )

\section{Characteristics of the Pulse Compression}

The seed amplification happens along with the pulse compression in the nonlinear regime. It means that the characteristics of the pulse compression give us much information about the amplification of the seed.
In order to investigate the characteristics of the pulse compression, we define two quantities, $D_{f}$ and $D_{r}$, which are the normalized half width of the seed as follows:

$$
\begin{aligned}
& D_{f}=\frac{\left|x_{s}-x_{\max }\right|}{\sigma_{i}}, \\
& D_{r}=\frac{\left|x_{\max }-x_{f, 1}\right|}{\sigma_{i}},
\end{aligned}
$$

where $x_{s}$ is the position of the starting point of the seed, $x_{\max }$ is the maximum point of the seed, and $x_{f, 1}$ is the first zero-crossing position of the normalized vector potential of the seed $\left(a_{s}\right) . \sigma_{i}$ is the initial FWHM of the seed.

Figure 2 shows the pulse broadening and compression in the front and rear half. Figure 2(a) shows the log-log plot of $D_{f}$ vs. time. The slope of these curves in compression phases does not very much. Figure 2(b) describes the log-log plot of $D_{r}$ vs. time. $D_{r}$ grows in $t^{\delta}$ in the broadening regime. After then, $D_{r}$ drops sharply at the start of the compression, and decreases as $t^{\eta}$. The sharp drop of $D_{r}$ comes from the local depletion of the seed.

The broadening and the compression behaviours appear in common throughout parameter ranges of the initial seed amplitude $a_{s 0}$, the initial pump amplitude $a_{p 0}$, and $\omega_{p}$ $/ \omega_{1}$. However, $D_{f}$ with variation of $a_{s 0}$ shows slightly different behaviours from those with the variation of other parameters.

Figure 2 shows $D_{f}$ and $D_{r}$ with different values of initial pump amplitude $a_{p 0}$. The larger $a_{p 0}$ results in the shorter broadening regime. For $D_{f}$, the slopes of the curve during the compression are the same for every $a_{p 0}$. The curve runs parallel to each other for different $a_{p 0}$ 's. The phenomenon is also shown for $D_{r}$ in Fig. 2(b). However, in case of $D_{r}$ curve, the slopes of the curves do not change during both the compression and the broadening phases. We can express these relationships as

$$
\begin{aligned}
& D_{f}=t^{-v}, \quad \text { (during compression) } \\
& D_{r}=t^{\delta} \text {, } \\
& D_{r}=t^{-\eta} \quad \text { (during compression) }
\end{aligned}
$$

where $v, \delta$, and $\eta$ were measured to be the same as 1.0 .

The difference between the behaviours of $D_{f}$ and $D_{r}$ comes from the first local depletion of the seed following that of the pump. The front part of the seed is not affected by the depletion, and there is no discontinuity in the derivative of $D_{f}$. At the rear part of the seed, there appear the zero-crossing points following the pump depletion. Emergence of the zero-crossing points is featured as the discontinuous derivative of $D_{r}$.

It takes some time until $D_{f}$ and $D_{r}$ follow the relationship explained in Eq. (4) because of transient behaviours. The relationship is satisfied when the seed is separated into two pulses and enters the nonlinear regime.

One more feature we should note in $D_{r}$ is the linear behaviour during pulse broadening. $D_{r}$ grows linearly with respect to time. It is connected with the counterpropagating pump with a constant velocity $v_{g, 0}$. As the pump evolves through the seed in the opposite direction, the rear end of the seed moves backward following the 
front end of the pump by stimulated RBS before the pump is totally depleted. It is the reason why $D_{r}$ in broadening regime grows linearly.

Figure 3 presents the time evolutions of $D_{f}$ and $D_{r}$ in terms of $\omega_{p} / \omega_{1}$. As $\omega_{p} / \omega_{1}$ becomes larger, the broadening regime becomes shorter and the compression starts earlier. In this case also, $v, \delta$, and $\eta$ become constant to be 1.0 with the variation of $\omega_{p} / \omega_{1}$.

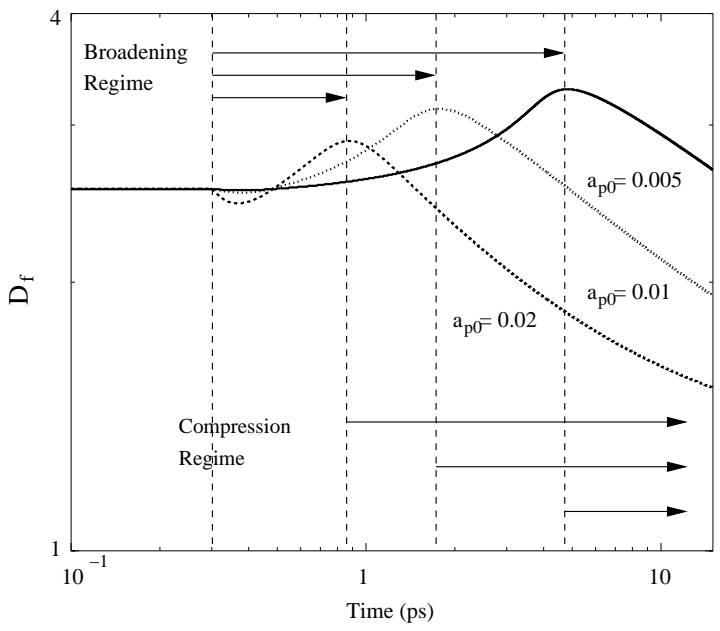

(a)

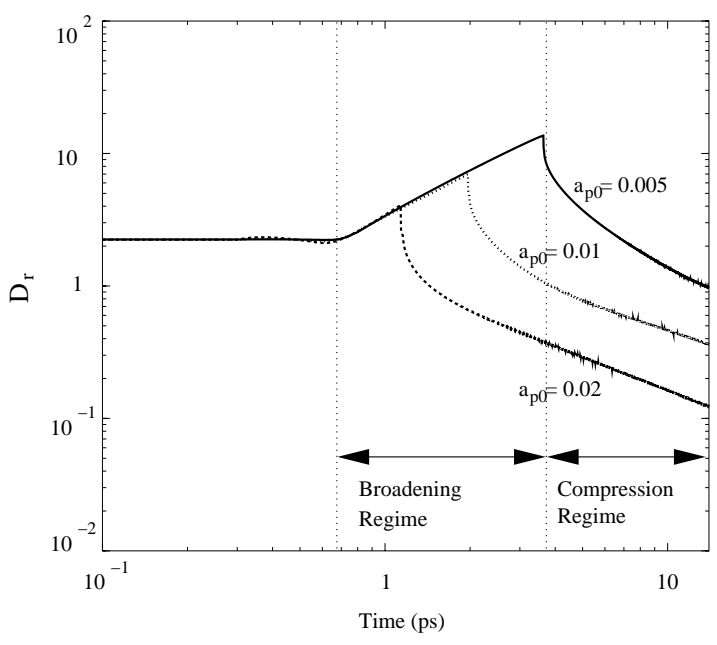

(b)

Figure 2: Broadening and compression regimes in (a) $D_{f}$ and (b) $D_{r}$ with the variation of initial pump amplitudes, $a_{p 0}\left(a_{s 0}=0.005, \omega_{p} / \omega_{1}=0.002, \tau_{p}=20 \mathrm{ps}, \tau_{s}=0.2 \mathrm{ps}\right)$

The variations of $a_{p 0}, a_{s 0}$, and $\omega_{p} / \omega_{1}$ are associated only with the starting time of compression. The larger $a_{p 0}, a_{s 0}$ and $\omega_{p} / \omega_{1}$ result in the shorter broadening regimes, the more seed amplification, and the more pulse compression under the same interaction length.

We observed that the exponents $v, \delta$, and $\eta$ are constant throughout the parameter ranges of the simulation. From our simulations, $v, \delta$, and $\eta$ are all equal to 1.0 in average. The fact is associated with that the growth rate of the peak energy in nonlinear regime is equal to 1.0. It means that the peak energy in nonlinear regime grows reciprocally to the compression of the seed. Therefore, the total energy proportional to the product of the peak intensity and the pulse width is saturated for the amplified seed pulse.

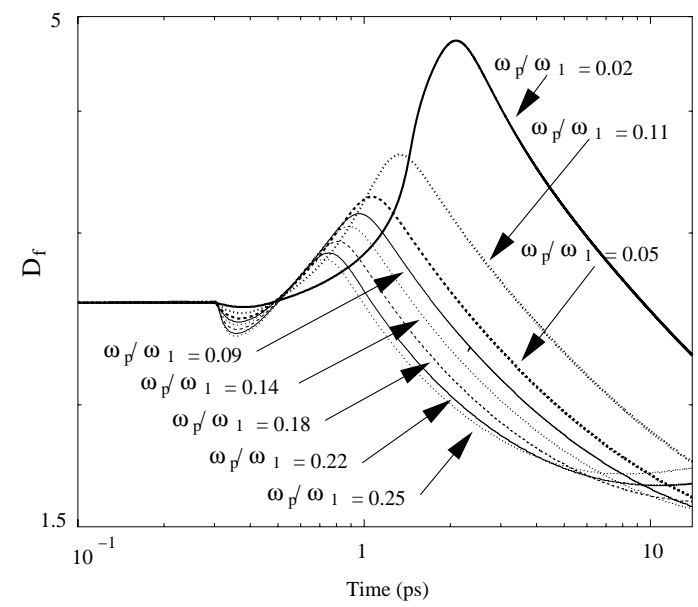

(a)

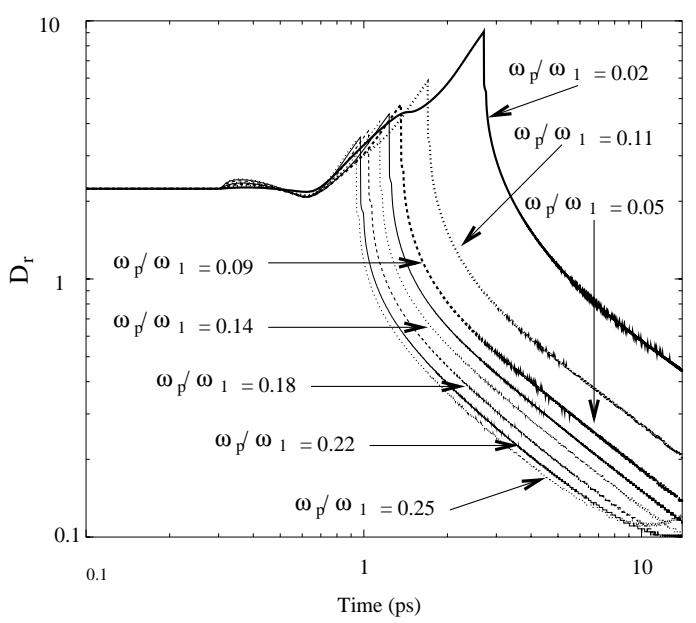

(b)

Figure 3: (a) $D_{f}$ and (b) $D_{r}$ curves in terms of different $\omega_{p} / \omega_{1}$ 's with the initial condition of $a_{p 0}=0.01, a_{s 0}=$ $0.001, \tau_{p}=20 \mathrm{ps}$, and $\tau_{s}=0.2 \mathrm{ps}$.

\section{REFERENCES}

[1] V. M. Malkin, G. Shvets, and N. J. Fisch, Phys. Plasma 7, No. 5, 2232, 2000.

[2] V. M. Malkin, G. Shvets, and N. J. Fisch, Phys. Rev. Lett. 82, No. 22, 4448, 1999.

[3] G. Shvets, N. J. Fisch, A. Pukhov and J. Meyer-terVehn, Phys. Rev. Lett. 81, No. 22, 4879, 1998.

[4] V. M. Malkin, G. Shvets, and N. J. Fisch, Phys. Rev. Lett. 84, No. 6, 1208, 2000.

[5] P. Mardahl, H. J. Lee, G. Penn, J. S. Wurtele, and N. J. Fisch, Phys. Lett. A 296, 109, 2002.

[6] H. J. Lee, P. J. Mardahl, G. Penn, and J. S. Wurtele, IEEE. Trans. Plasma. Sci. (Special Issue) 30, No. 1, 2002. 